\title{
Lessons Learned and Future Directions: CLER National Report of Findings 2016
}

\author{
Robin Wagner, RN, MHSA and Kevin B. Weiss, MD
}

The Clinical Learning Environment Review (CLER) Program is designed to provide formative feedback to the hospitals, medical centers, and ambulatory care sites that serve as clinical learning environments (CLEs) for ACGME-accredited residency and fellowship programs. ${ }^{1,2}$ While its main focus is to provide institutions with individual feedback, the aggregate data presented here offer an important overview of the environments where residents and fellows train-and thus can inform the national conversation on optimal attributes for a CLE. The collective observations from this first set of CLER site visits portray a community of teaching hospi"The collective observations from this first set of CLER site visits portray a community of teaching hospitals, medical centers, and ambulatory care sites that has great capacity to shape the quality of the emerging physician workforce and drive improvements in patient care." tals, medical centers, and ambulatory care sites that has great capacity to shape the quality of the emerging physician workforce and drive improvements in patient care.

These 297 initial visits sought to establish baseline structural characteristics of the clinical sites, as well as their training practices in the six focus areas for residents and fellows. In future cycles, the CLER Program will also seek to understand how the sites identify and prioritize areas for improvement and assess progress over time.

While the aggregated data from this first set of CLER visits yielded many findings specific to the six focus areas, several overarching themes also emerged. Three of them relate to the first question CLER sought to answer, ${ }^{3}$ namely: "What is the clinical learning environment's infrastructure for addressing the six focus areas?" The themes that responded to this question noted that CLEs vary in:

- their approaches to and capacity for improving patient safety and health care quality and the degree to which they engage residents and fellows in addressing these areas,

- the nature of their strategic relationships with graduate medical education (GME), and

- the degree to which they coordinate educational resources across the organization.

The fourth overarching finding addressed how CLEs relate to their associated GME communities. The data show that CLEs vary in their approach to engaging GME faculty members, including program directors, and the degree to which they invest in faculty education and training in patient safety, health care quality, and the other focus areas. In addition, while not formally queried about workload, many faculty members volunteered that their expanding duties were adversely affecting their capacity to teach and oversee residents and fellows.

In keeping with its model of continuous quality improvement, the CLER Program has now begun its second set of visits to CLEs with three or more ACGME-accredited core specialty programs. The site visit protocol has been updated to closely align with the CLER Pathways to Excellence recommendations, ${ }^{4}$ and in particular its observation that faculty members must serve as role models for residents and fellows. The second set of CLER visits will explore faculty engagement in greater detail, including whether they are modeling the desired behaviors in the six focus areas.

In time, the CLER Program will also seek to identify overarching themes associated with outcomes, in particular those related to resident and fellow engagement in the six focus areas. The first set of CLER visits showed that, while residents and fellows participate in educational activities related to the focus areas, the degree of experiential learning and active engagement varies both within and across sites. Thus, in the future, CLER will seek to clarify whether this variation might affect important outcomes like patient care or resident and fellow training. 


\section{Future Directions}

The CLER Program has begun a journey of exploring and improving the quality of patient care by seeking to better understand the CLEs in which residents and fellows develop the skills necessary for independent practice.

Over the coming year, the CLER Program will continue that journey by sharing additional data through a series of issue briefs, white papers, conferences, workshops, and other educational events. In addition, the program will develop and pilot new sub-protocols that focus on the surgical environment and on patient experience as it relates to resident and fellow learners. These sub-protocols will explore how the governing bodies of CLEs conceive and manage the educational strategies of their GME mission.

In shaping the CLER Program, the CLER Evaluation Committee will continue to review all aspects of the program, including voluntary responses to the site visit reports as well as new data sources (e.g., post-visit experience, focus groups) developed and implemented to guide the program.

The CLER Evaluation Committee and the ACGME Board of Directors will also re-evaluate the program's six areas of focus periodically, recognizing that these areas examine only a portion of the full context of a CLE.

Suggestions for additional future areas of exploration include a more in-depth focus on resident, fellow, and faculty wellness; additional exploration of interprofessional learning, teamwork, and collaborative practice; and evaluating how CLE/GME integration can further the Institute for Healthcare Improvement's "triple aim" of achieving better care for patients, better health for communities, and lower costs. ${ }^{5}$

Since 2012, the CLER Program has periodically convened national conversations with key organizations that share a common interest in improving the CLE. In 2015 , this group created a formal identity, charter, and set of goals. It is now known as the National Collaborative for Improving the Clinical Learning Environment (NCICLE) and will continue to contribute to the evolution of the CLE.

"The CLER Program, along with other ACGME programs, will continue to explore the variation in practices that the CLER site visits discovered both within and across CLEs in the six focus areas."

Lastly, the CLER Program, along with other ACGME

programs, will continue to explore the variation in practices that the CLER site visits discovered both within and across CLEs in the six focus areas. Some of this variability is likely useful while other variability may be either unnecessary or counter-productive to excellence.

To help identify which practices are best for both GME and patient care, the ACGME recently launched a new four-year initiative called Pursuing Excellence in Clinical Learning Environments. This initiative sets up a collaborative, peer-to-peer shared learning system based on the goals outlined in the CLER Pathways to Excellence document. ${ }^{6}$ Recognizing that there are no simple answers to achieving excellence in the CLE, the ACGME has allocated resources to support explorations in the six areas and help facilitate sharing of successful practices throughout the CLE community. Over time, the organizations involved in Pursuing Excellence will contribute substantially to the growing body of resources for assisting CLEs on the path towards excellence. These collective efforts both within the CLER Program and in alignment with other national organizations seek to improve the quality of GME and patient care in all CLEs.

\footnotetext{
${ }^{1}$ Weiss KB, Wagner R, Nasca TJ. Development, testing, and implementation of the ACGME Clinical Learning Environment Review (CLER) Program. J Grad Med Educ. 2012;4(3):396-398.

${ }^{2}$ Weiss KB, Bagian JP, Nasca TJ. The clinical learning environment: the foundation of graduate medical education. JAMA. 2013;309(16):1687-1688.

${ }^{3}$ Wagner R, Patow C, Newton R, Casey BR, Koh NJ, Weiss KB; CLER Program. The overview of the CLER Program: CLER National Report of Findings 2016. J Grad Med Educ. 2016;8(2 suppl 1):11-14.

${ }^{4}$ The Accreditation Council for Graduate Medical Education (ACGME). CLER Pathways to Excellence: expectations for an optimal clinical learning environment to achieve safe and high quality patient care. ACGME website. https://www. acgme.org/acgmeweb/Portals/0/PDFs/CLER/CLER_Brochure.pdf. Accessed January 25, 2016.

${ }^{5}$ Berwick DM, Nolan TW, Whittington J. The triple aim: care, health, and cost. Health Aff (Millwood). 2008;27(3):759-769. ${ }^{6}$ Wagner R, Weiss KB, Passiment ML, Nasca TJ. Pursuing excellence in clinical learning environments. J Grad Med Educ. 2016;8(1):124-127.
} 